\title{
Molecular imprinting: A new tool for drug innovation
}

\section{Cornelus F. van Nostrum}

Department of Pharmaceutics, Utrecht Institute for Pharmaceutical Sciences, Utrecht University, P.O. Box 80082, 3508 TB Utrecht, The Netherlands

\begin{abstract}
Molecular imprinting is a rapidly evolving technique to prepare synthetic receptors. This paper discusses the opportunities to use such synthetic receptors for pharmaceutical applications, including separation, extraction and detection of drugs or their metabolites, and methods for drug screening, drug delivery and drug targeting. Molecularly imprinted polymers can be a valuable alternative for receptors of biological origin, such as antibodies, in terms of robustness, versatility and ease of preparation.
\end{abstract}

\section{Introduction}

Molecular recognition is defined as "the ability of one molecule to attach to another molecule that has a complementary shape" (http://www.wordreference.com). Evolution has provided biology with many intriguing examples of molecular recognition, including those involved in interactions between a ligand and a receptor (such as substrate and enzyme, antigen and antibody), and in transport and signal transduction processes. Moreover, the action of the majority of drugs relies on the phenomena of molecular recognition. Binding events in biology are usually characterized by high selectivity and high association constants in the order of $10^{3}$ to $10^{12} \mathrm{~L} \mathrm{~mol}^{-1}$.

Mankind has succeeded in preparing synthetic receptors for a variety of applications [1], sometimes in the effort to mimic or even to overcome nature. Mostly, synthetic receptors are designed by the chemist, often with computational aid using molecular modeling, and are subsequently synthe-

E-mail address: C.F. van Nostrum (c.f.vannostrum@pharm.uu.nl) URL: http://www.pharm.uu.nl/pharmaceutics

\section{Section Editors:}

Daan J.A. Crommelin - Department of Pharmaceutical Sciences, Utrecht University, Utrecht, The Netherlands Gerrit Borchard - Enzon Pharmaceuticals, Piscataway, NJ, USA

sized in the laboratory. This approach is characterized by the assembly of carefully chosen functional groups in a receptor molecule in a predefined way so that they can optimally interact with the ligand of interest. Although this approach is rather tedious, it can sometimes afford receptors with very high affinity constants and good selectivity. Another approach relies on the self-assembly and subsequent fixation of functional groups around a template molecule. The latter approach is called "molecular imprinting".

\section{Key technologies}

Molecular imprinting involves the preparation of a solid material (usually a synthetic polymer) containing cavities that have a shape and functional groups complementary to the imprinted template molecule (see Box 1). Traditionally, there are two different ways to make a molecular imprinted polymer (MIP), respectively, pioneered by Wulff and Schauhoff [2] and Mosbach and co-workers [3] and involves covalent or non-covalent binding of the template to the monomer in the first step of the MIP synthesis. With both strategies, MIPs should be able to recognize the imprinted molecule, or a structurally related molecule, in a selective way. The binding affinity can be moderate or high, depending on the application that is aimed for. The non-covalent approach has the advantage of being more versatile and is currently the most frequently used method.

In contrast to biological receptors, which usually have one or more well-defined binding sites showing a single associa- 


\section{Box I. Molecular imprinting}

(a)

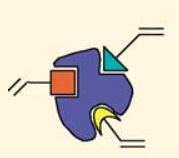

(b)

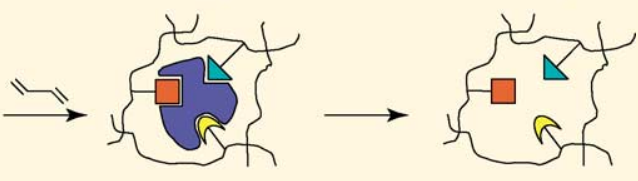

Drug Discovery Today: Technologies

The process of preparing a molecularly imprinted material involves three main steps: (a) the mixing or binding of one or several (functional) monomers with the template molecule (printed in blue) in a suitable solvent or dispersion liquid; (b) the polymerization of monomers in the presence of a cross-linker to prepare a polymer network in which the functional monomers become fixed around the template and (c) removal of the template from the solid.

tion constant $\left(K_{\mathrm{a}}\right)$, MIPs are usually characterized by their binding site heterogeneity, and therefore, a distribution of $K_{\mathrm{a}}$ values. This is mainly caused because the binding of the template to the functional monomer(s) (i.e., in the first step of the preparation of an MIP using the non-covalent approach, see Box 1) is an equilibrium process. Depending on the equilibrium constants, there will be several functional monomers not bound to the template molecule, which will result in the formation of non-optimal binding sites in the polymer. The best way to overcome this problem, and which has shown to be successful in many cases including drug imprinting [4], is to use the so-called stoichiometric binding between template and functional monomers. In this approach, a functional monomer is selected, which has such high affinity for the template that there are hardly any unbound functional monomers present in the polymerization mixture.

Another common problem in the field of molecular imprinting is the incomplete removal of the template and slow binding kinetics of the ligand. This is especially a problem when large template molecules are involved, for example, proteins. Improvements have been made recently, for example, by confining the preparation of imprints to the surface (or interface) of a material [5-7] or utilizing materials with a well-defined porosity (e.g., silica) as a solid support for the MIP [8]. In another approach to optimize protein imprinting, Rachkov and Minoura have introduced the concept of epitope imprinting, viz. the use of a small part of a protein to be used as the template to allow binding of the complete protein to the MIP [9]. The company Aspira Biosystems (http://www.aspirabio.com, Table 1) has commercialized such an epitope concept using a technology which they call partially molecular imprinting (PMI) [10]. Aspira is applying this technology by making arrays of protein capture agents for detecting specific proteins in biological samples.

The issues mentioned above clearly illustrate that molecular imprinting is a technology that is growing beyond its infancy and is clearly developing; more and more applications have emerged in recent years. Typically, as will be shown below, MIPs are frequently mentioned in the context of antibody substitutes in a variety of applications. The term "artificial antibody" is sometimes used as a synonym for an MIP. Whether this is justified will be discussed after the following overview, which is related to the (possible) applications of MIPs in the analysis, development and delivery of drugs.

\section{Separation and extraction}

From the beginning, molecular imprinting has found its main application in the field of chromatography and solid phase extraction (SPE) for the separation, pre-concentration and detection of low molecular weight compounds, including drugs and drug metabolites from, for example, blood plasma and urine samples. One of the major hurdles that had to be taken into account was the fact that sustained template leakage can interfere with the detection of the analyte. Nowadays, this technology is the most matured and widely commercialized application of MIPs. For example,

Table I. Molecularly imprinted polymers in commercial products

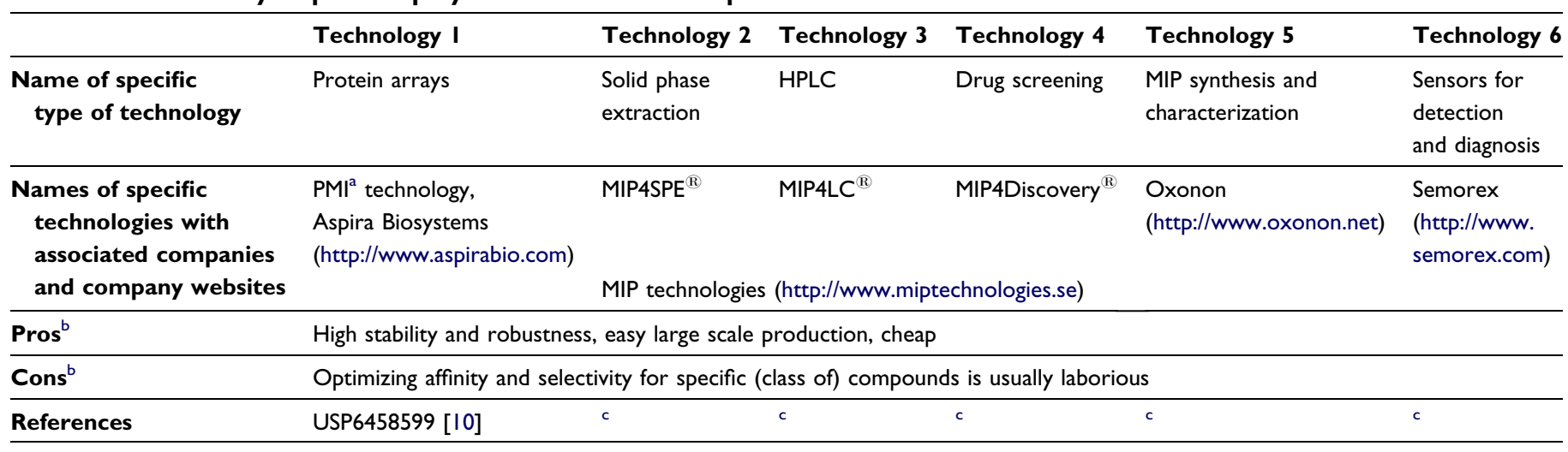

${ }^{a}$ Partial molecular imprinting.

${ }^{\mathrm{b}}$ Advantages and disadvantages are typical for all MIP-based technologies.

${ }^{\mathrm{c}}$ No patents could be identified that are specific for each technology and are assigned to the companies mentioned. 
MIP technologies (http://www.miptechnologies.se, Table 1) is a company with technology entirely based on MIPs, with its main product lines focusing on compound extraction (MIP4SPE ${ }^{\mathbb{R}}$, molecularly imprinted polymers for solid phase extraction) and separation (MIP4LC ${ }^{\mathbb{R}}$, for HPLC). Enantiomeric separation of chiral molecules by HPLC has proven possible with MIPs as the stationary phase [11], and even separation of proteins with great structural similarities (viz. horse myoglobin versus whale myoglobin) has been reported [12]. Classical SPE and chromatographical methods are generally based on generic physico-chemical properties of the analyte and the stationary phase, whereas MIPs can surpass the selectivity of those classical materials, because adsorption to MIPs can rely on more subtle structural differences between molecules. In terms of selectivity, MIPs can be compared to immunoaffinity extraction/separation, where antibodies that are covalently bound to a solid support are used. The most important advantages of MIPs are the increased robustness and low cost of the materials compared with antibody-based separation.

An interesting pharmacological application of MIPs that closely resembles SPE for analysis, is the in vivo use of MIPs as a "molecular trap" to adsorb substances, such as glucose, cholesterol, bile acids or caffeine, for example, from the gastrointestinal tract thereby preventing their adsorption by the body. For example, a patent has been issued recently on MIPs to trap bile acids, with improved selectivity as compared with other bile acid adsorbants, such as cholestyramine [13].

\section{Ligand binding assays and sensors}

Immunoassays are typically used to determine trace amounts of substances from biological samples such as human serum. Again, because of the limitations and costs that are associated with the use of antibodies, molecularly imprinted sorbent assays could be very useful alternatives. MIPs made against theophylline and diazepam showed strong binding and crossreactivity profiles similar to those of antibodies and have been used in a radiolabelled ligand-binding assay by the group of Mosbach and co-workers [14]. The sensitivity and accuracy was comparable to the results obtained by a traditional radioimmunoassay technique. Again, the robustness of an MIP assay surpasses that of an antibody-based assay. The main challenges that are currently being tackled are the reduction of nonspecific adsorption to the polymer, and the development of other detection methods than using radiolabels, such as scintillation proximity assays, ELISA-type assays, or assays using fluorescent or electroactive probes (for a recent overview, see: Ye and Haupt, 2004, in the list of related articles).

The possibility to detect the binding between an analyte and an MIP by transduction into a specific signal has led to the development of MIP sensors. Detection methods include measurement of mass change, field-effect capacitance, or conductivity, fluorescence-based detection methods, and so forth. Several excellent reviews on this subject can be found in literature $[15,16]$ (also see, Ye and Haupt, 2004, in the list of related articles).

\section{Drug screening}

Looking at MIPs as artificial receptors (antibody-mimics), one can imagine that a polymer imprinted with a specific ligand can be used to screen a combinatorial library to identify compounds that closely match the binding strength of the imprinted ligand. This concept has been demonstrated to be feasible indeed $[17,18]$, and has been commercialized now by the previously mentioned company MIP technologies (MIP4Discovery ${ }^{\mathbb{R}}$ ). Screening of a library can be done by evaluating the retention times in a HPLC setup using the MIPs as a stationary phase. Thus, the low cost, high stability and relative ease of preparation of MIPs can facilitate the primary screening for alternative substances (agonists or antagonists) that bind to the biological receptor of the known ligand, especially when the biological receptor itself is not readily available. Therefore, molecular imprinting has the potential to play an important role in drug development in the future.

\section{Drug delivery}

Solid polymers or hydrogels are frequently chosen as a material to allow controlled delivery of drugs. Drug release might be governed and controlled by diffusion, degradation of the matrix, or a combination of both. There is an ongoing interest to identify additional tools to modify the release profile of a drug from a polymer matrix, and molecular imprinting has been suggested as one of those tools.

Two comprehensive review papers have been recently published about the use of MIPs in drug delivery applications: see Alvarez-Lorenzo and Concheiro (2004) and Hilt and Byrne (2004) in the list of related articles. Therefore, the present contribution will only provide a birds-eye view of the recent developments in this field. Fig. 1 summarizes the various possibilities.

The fact that an MIP has a certain affinity for an imprinted drug has been exploited to enhance the loading capacity of the polymer. For instance, Hiratani et al. have significantly improved loading of timolol in an imprinted hydrogel with respect to a non-imprinted polymer for ophthalmic delivery from soft contact lenses [19]. However, because the drug is required as the template and, as a consequence, the gels are already loaded with the drug during preparation, practical use will be in the efficient reloadability of the delivery system. In addition, the molecular imprinting effect has been used for sustained release of a drug from an MIP. This was the case for the timolol-imprinted soft contact lenses and has been shown by others as well, for example, by Allender $e t a l$. with the release of propranolol from a propranolol-imprinted transdermal delivery system [20]. Enantioselective release 


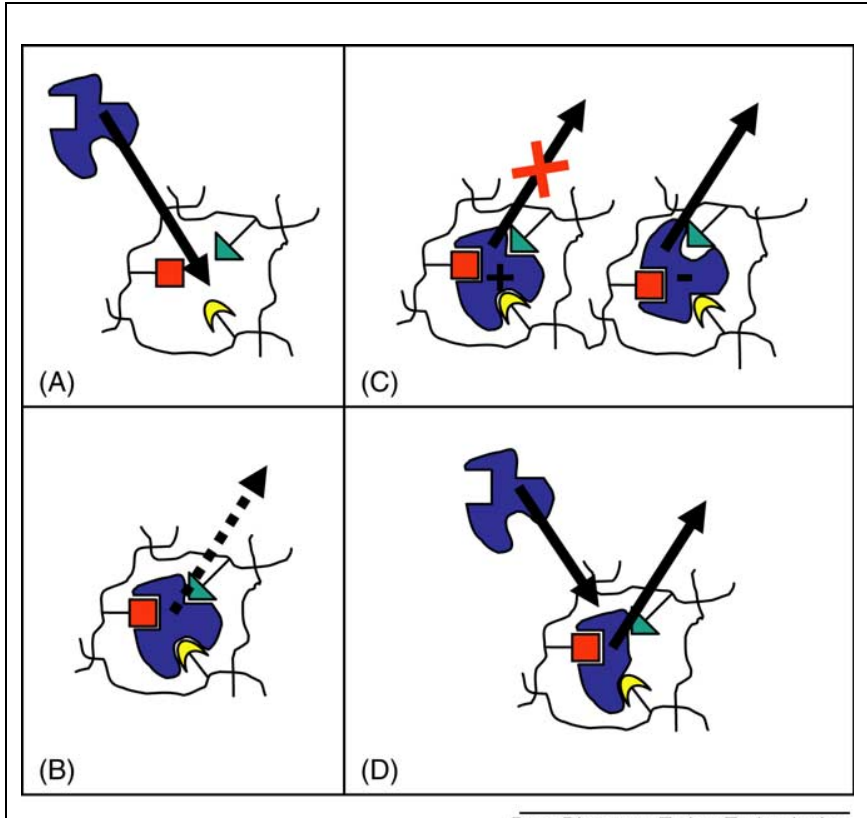

Drug Discovery Today: Technologies

Figure I. Modes of modifying drug loading and release by molecular imprinting. Panel A (enhanced reloading): the binding sites present in the MIP allow a high loading capacity for the imprinted drug. Panel B (sustained release): imprinted drug molecules are released slowly because of their high affinity to the MIP. Panel C (enantioselective release): the imprinted enantiomer $(+)$ is retained in the MIP, whereas the non-imprinted enantiomer $(-)$ does not fit properly in the binding site and will be released. Panel $D$ (responsive release): a drug with low affinity is replaced by an imprinted stimulus that has a higher affinity for the binding site. The imprinted binding sites in each panel are represented by the functional groups printed in three different colours (see Box I); the drug molecules are shown in blue.

has been reported in a few cases, that is, a polymer imprinted with one enantiomer is able to release the same enantiomer with a slower rate than the opposite enantiomer from a matrix loaded with the racemic drug [21]. However, optimal selectivity (viz. release of the non-imprinted enantiomer with full retention of the imprinted enantiomer) has not been reached yet. Moreover, pure enantiomers are still required as the template for the preparation of the MIP, which also hampers the practical benefit of this idea.

An interesting and potentially useful application of MIPs in drug delivery is the responsive drug release based on competitive binding. This is achieved with a polymer that releases a weakly bound drug stimulated by binding of the imprinted compound (Fig. 1). Sreenivasan was the first to describe this concept by showing the release of testosteron induced by hydrocortison from a hydrocortison-imprinted polymer [22].

\section{Drug targeting}

As mentioned before, many researchers are inspired by the fact that MIPs can be used as antibody-mimics. One application of antibodies in the pharmaceutical field that has not been mentioned so far, is their use in drug targeting. Being a moiety that can selectively recognize (pathogenic) cells or tissues, antibodies have been used as drug targeting devices coupled to drug carriers, such as liposomes [23] or polymeric micelles [24]. Therefore, in principle, a synthetic drug targeting device could be designed by creating imprints of targets that are exposed on cell or tissue surfaces, such as oligosaccharides, glycoproteins, or membrane proteins (Fig. 2) [25]. The fact that this idea has hardly been worked upon indicates its complexity. The hurdles that have to be taken to accomplish a useful in vivo MIP targeting device are manifold (see Outstanding issues). However, the great progress that is currently made in the field of molecular imprinting will probably make this attractive goal feasible in the future.

\section{Conclusions}

MIPs are frequently considered as artificial antibodies and many technologies mentioned in this review are based on the use of MIPs as antibody-mimics. Therefore, a comparison of the typical features of a natural antibody and an MIP is useful, if one wishes to evaluate whether MIPs will have a great future as a generic antibody replacement strategy for pharmaceutical applications. Table 2 lists several characteristics of both the receptor systems.

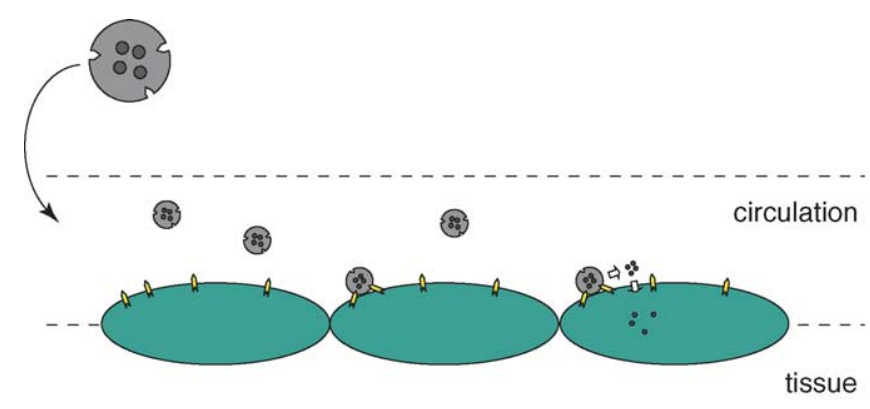

Drug Discovery Today: Technologies

Figure 2. Suggested method to achieve in vivo targeting through molecular imprinting. Drug loaded nanoparticles (gray) adhere to imprinted cellular receptors (yellow) and deliver their contents to the cells (green). 
Table 2. Antibodies versus molecularly imprinted polymers (MIP)

\begin{tabular}{lll}
\hline & MIP & Antibody \\
\hline Affinity & $\begin{array}{l}10^{3} \text { to } 10^{9} \mathrm{~L} \mathrm{~mol}^{-1} \\
\text { (heterogeneous) }\end{array}$ & $\begin{array}{l}5 \times 10^{4} \text { to } 10^{12} \mathrm{~L} \mathrm{~mol}^{-1} \\
\text { (homogeneous) }\end{array}$ \\
\hline Selectivity & Medium-high & High \\
\hline Stability & High & Low \\
\hline Production & $\begin{array}{l}\text { Easy, cheap, } \\
\text { large scale }\end{array}$ & $\begin{array}{l}\text { Laborious, expensive, } \\
\text { small scale }\end{array}$ \\
\hline
\end{tabular}

MIPs show affinities for the imprinted compound that are reasonably high and mostly in the same range of the binding strengths between an antibody and its antigen, but the heterogeneity of the binding sites can sometimes be a problem that is, however, solvable. Another concern is that the selectivity of MIPs is still a limiting factor for some applications, although similar or sometimes even higher selectivities have been reported when compared with natural antibodies.

The major benefits of MIPs compared with antibodies are their high - and almost unlimited - stability and their easy way of preparation at a large scale that unquestionably outperform antibodies in terms of costs. Applications where these features are the limiting factors in the development of antibody-based technologies are the most interesting to explore. MIPs can nowadays be prepared for almost any kind of compound, including large biomolecules, such as proteins and oligonucleotides but it mostly requires a lot of optimization effort to reach the optimal binding efficiency and selectivity for each target compound. Combinatorial approaches, high throughput screening and computational aid can help a lot in that respect.

Relatively unexplored areas where MIPs can become a cheap and good alternative for antibodies are drug discovery and drug targeting, the latter being a particularly challenging application. Besides, molecular imprinting is a valuable tool in the development of sustained and stimulus-responsive drug release systems. We might expect many new developments emerging in these fields in the near future.

\section{Related articles}

Alvarez-Lorenzo, C. and Concheiro, A. (2004) Molecularly imprinted polymers for drug delivery. J. Chromatogr. B 804, 231-245 Andersson, L.I. (2000) Molecular imprinting for drug bioanalysis. A review on the application of imprinted polymers to solid-phase extraction and binding assay. J. Chromatogr. B 739, 163-173 Hilt, J.Z. and Byrne, M.E. (2004) Configurational biomimesis in drug delivery: molecular imprinting of biologically significant molecules. Adv. Drug Deliv. Rev. 56, 1599-1620

Ye, L. and Haupt, K. (2004) Molecularly imprinted polymers as antibody and receptor mimics for assays, sensor and drug discovery. Anal. Bioanal. Chem. 378, 1887-1897

\section{Outstanding issues}

Drug targeting by molecular imprinting requires the following points for consideration:

- Imprints have to be readily accessible for the cellular target (i.e., at the surface of the MIP);

- The cellular targets are often large biomolecules that are difficult to imprint;

- MIPs should be in the form of small and biocompatible nanoparticles to allow free flowing in, for example, the circulation;

- High affinities are required which are difficult to achieve in an aqueous environment.

These issues have been addressed more or less individually with some success, but the development of MIPs with a combination of all these features still remains an interesting challenge.

\section{References}

1 Schrader, T. and Hamilton, A.D. (2005) Functional Synthetic Receptors. Wiley

2 Wulff, G. and Schauhoff, S. (1991) Enzyme-analog-built polymers. 27. Racemic-resolution of free sugars with macroporous polymers prepared by molecular imprinting - selective dependence on the arrangement of functional groups versus spatial requirements. J. Org. Chem. 56, 395-400

3 Sellergren, B. et al. (1988) Highly enantioselective and substrate-selective polymers obtained by molecular imprinting utilizing noncovalent interactions - NMR and chromatrographic studies on the nature of recognition. J. Am. Chem. Soc. 110, 5853-5860

4 Lubke, C. et al. (2000) Imprinted polymers prepared with stoichiometric template-monomer complexes: efficient binding of ampicillin from aqueous solutions. Macromolecules 33, 5098-5105

5 Yilmaz, E. et al. (2000) The use of immobilized templates - a new approach in molecular imprinting. Angew. Chem. Int. Ed. 39, 2115-2118

6 Kempe, M. et al. (1995) An approach towards surface imprinting using the enzyme ribonuclease A. J. Mol. Recogn. 8, 35-39

7 Shi, H.Q. et al. (1999) Template-imprinted nanostructured surfaces for protein recognition. Nature 398, 593-597

8 Sulitzky, C. et al. (2002) Grafting of molecularly imprinted polymer films on silica supports containing surface-bound free radical initiators. Macromolecules 35, 79-91

9 Rachkov, A. and Minoura, N. (2001) Towards molecularly imprinted polymers selective to peptides and proteins. The epitope approach. Biochim. Biophys. Acta 1544, 255-266

10 Huang, C.S. [Aspira Biosystems] (2002) Compositions and methods for capturing, isolating, detecting, analyzing and quantifying macromolecules. USP6458599

11 Kandimalla, V.B. and Ju, H.X. (2004) Molecular imprinting: a dynamic technique for diverse applications in analytical chemistry. Anal. Bioanal. Chem. 380, 587-605

12 Hjertén, S. et al. (1997) Gels mimicking antibodies in their selective recognition of proteins. Chromatographia 44, 227-234

13 Green, B.S. and Priwler, M. (2004) Molecularly imprinted polymers for the treatment and diagnosis of medical conditions. US2004062745

14 Vlatakis, G. et al. (1993) Drug assay using antibody mimics made by molecular imprinting. Nature 361, 645-647

15 Al-Kindy, S. et al. (2000) Molecularly imprinted polymers and optica sensing applications. Crit. Rev. Anal. Chem. 30, 291-309

16 Haupt, K. and Mosbach, K. (2000) Molecularly imprinted polymers and their use in biomimetic sensors. Chem. Rev. 100, 2495-2504

17 Ye, L. et al. (2001) Towards the development of molecularly imprinted artificial receptors for the screening of estrogenic chemicals. Analyst 126, $760-765$ 
18 Vallano, P.T. and Remcho, V.T. (2000) Affinity screening by packed capillary high-performance liquid chromatography using molecular imprinted sorbents I. Demonstration of feasibility. J. Chromatogr. A 888, 23-34

19 Hiratani, H. et al. (2005) Ocular release of timolol from molecularly imprinted soft contact lenses. Biomaterials 26, 1293-1298

20 Allender, C.J. et al. (2000) Pharmaceutical applications for molecularly imprinted polymers. Int. J. Pharm. 195, 39-43

21 Suedee, R. et al. (2000) Evaluation of matrices containing molecularly imprinted polymers in the enantioselective controlled delivery of $\beta$ blockers. I. Control. Release 66, 135-147
22 Sreenivasan, K. (1999) On the application of molecularly imprinted poly(HEMA) as a template responsive release system. J. Appl. Polym. Sci. 71, 1819-1821

23 Mastrobattista, E. et al. (1999) Immunoliposomes for the targeted delivery of antitumor drugs. Adv. Drug Deliv. Rev. 40, 103-127

24 Torchilin, V.P. (2004) Targeted polymeric micelles for delivery of poorly soluble drugs. Cell Mol. Life Sci. 61, 2549-2559

25 Nagahori, N. and Nishimura, S.I. et al. (2001) Tailored glycopolymers: controlling the carbohydrate-protein interaction based on template effect. Biomacromolecules 2, 22-24 